\title{
EXPERIMENTAL STUDIES OF TRIPLET EXCITON BANDS OF MOLECULAR CRYSTALS†
}

\author{
R. M. Hochstrasser,+ T.-Y. Li, H.-N. Sung, J. Wessei. and A. H. Zewail. \\ Department of Chemistry and Laboratory for Research on the Structure of \\ Matter, University of Pennsylvania, Philadelphia, Pennsylvania 19174, USA
}

\begin{abstract}
Methods of studying properties of triplet exciton states of organic crystals are presented with an emphasis on exposing the dimensionality of excitons. Results of isotopic replacement spectra for 1,4-dibromonaphthalene which is a linear chain, and for halogenated benzenes, which are likely to be linear chains, are presented. Luminescence studies of linear chain exciton systems are shown to yield information about the stationary states of small clusters. Finally some preliminary studies of two-photon spectra of naphthalene excitons are described.
\end{abstract}

Experiments have now established a number of characteristic propertics for triplet excitons in organic crystals

(i) Triplet exciton bandwidths are often small in the range $c a .0$ to $40 \mathrm{~cm}^{-1}$. An exception appears to be diiodobenzene ${ }^{1}$ where the band maybe $c a$. $10^{3} \mathrm{~cm}^{-1}$. Since all cases studied except diiodobenzene are clearly in the weak coupling ${ }^{2}$ limit, we use the term bandwidth with reference to a particular vibronic state- - often the zero-point level of the triplet state. The bandwidth is not exposed by observing a factor group splitting since the translationally equivalent interactions may dominate.

(ii) The theoretical prediction ${ }^{3}$ that short-range resonance force interactions should determine the band structure of triplet excitons is experimentally verified for the case of dibromonaphthalene ${ }^{4}$. The implication is that the energy transfer topology depends on intermolecular overlap and that there is a possibility of predicting band structure and energy transfer anisotropy from crystal structure.

(iii) The phonon sidebands associated with singlet-triplet transitions in crystals are often strong and may even be stronger than the zero phonon lines. This condition arises when the bandwidths are very small ${ }^{5}$ since there may then be many lattice motions during the jump time of the electronic excitation.

One of the most interesting aspects of triplet excitations in molecular crystals concerns the relationships between the energy transfer topology-the

+ This research was supported in part by the NSF-MRL contract No. GH-33633. in part by a Public Health Grant GM12592, and in part by an NSF grant.

$¥$ Presenter of paper at Tallinn 
effective dimensionality of the exciton-and the chemical and crystal structure of the system. Experience with anthracene ${ }^{6}$ and naphthalene ${ }^{7}$ showed that approximate numerical calculations ${ }^{3}$ were not reliable in predicting details of band structure. and a real need for experiments on a wider class of molecules became apparent. Our starting point was a study of crystals that display no Davydov splitting of their singlet-triplet transition. Excitons in such systems may have either a simple dispersion similar to that in a crystal having one molecule per unit cell. or each of the resonance interactions must be much smaller than the optical line width. In the simplest three dimensional case the dispersion will be given by:

$$
\varepsilon_{k}=\Sigma 2 V_{x} \cos (\boldsymbol{r}, \boldsymbol{k})
$$

where $V_{x}$ is the nearest neighbour resonance force interaction. For the dispersion (equation 1) to occur in a real crystal the interactions between molecules in the different translational stacks (labelled by $a$ ) must be negligible: this is a feature of the restricted Frenkel limit ${ }^{8}$. We have shown ${ }^{4}{ }^{4}$ that 1.4-dibromonaphthalene (DBN) is a linear chain with $V_{a}=V_{b}=0$ and

Table 1. Structural data for some halogenated aromatics.

\begin{tabular}{|c|c|c|c|c|c|}
\hline & $a(\AA)$ & $b(\AA)$ & $c(\mathbb{A})$ & $z$ & $\beta$ \\
\hline$\alpha$-p-dichlorobenzene ${ }^{\dagger}$ & 14.63 & 5.66 & 3.89 & 2 & 113.3 \\
\hline$p$-dibromobenzene + & 15.36 & 5.75 & 4.10 & 2 & 112.5 \\
\hline sym-trichlorobenzene $\$$ & 13.77 & 13.07 & 3.83 & 4 & 90 \\
\hline sym-tribromobenzeneş & 13.93 & 13.19 & 3.91 & 4 & 90 \\
\hline sym-tetrachlorobenzene $\|$ & 9.60 & 10.59 & 3.76 & 2 & $102.5(92.5)$ \\
\hline B-sym-tetrabromobenzene & 10.32 & 10.71 & 4.02 & 2 & 102.3 \\
\hline hexachlorobenzenett & 8.08 & 3.87 & 16.65 & 2 & 117.0 \\
\hline
\end{tabular}

\footnotetext{
+ E. Frasson. C. Garbuglio and S. Bezri, Acta Cryst.., 12. 126 (1459).

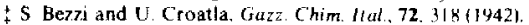

$\$$ H. J Milledge and L. M. Paut. Acta Cryst. 13. 2 (95 (1960).

If r. H. Herbstein. Acra Crist. 18.997 1965 .

- (j. Ganner and F. H. Herbstein. Acta Cryst. 13, 706 11960)

I. N. Strel'tsova and Y. I. Struchkor. J Struct. Chem. 2. $296(1961)$.
}

$V_{c}=7.4 \pm 0.4 \mathrm{~cm}^{-1}$ for the 0.0 -level of the first triplet state. A number of similar systems studied in this laboratory are shown in Table $l$ and it is seen that the crystals of halogenated aromatics have the common feature of one very short intermolecular contact. It is reasonable to guess that they are all essentially linear chain exciton systems and the first part of this paper will be a summary of optical experiments designed to expose the exciton band structures of some of these materials.

\section{1,4-Dibromonaphthalene}

Whiteman's experiments ${ }^{+}$can be understood from the scheme shown in Figure $I$. When the crystal is neat. optical transitions occur only to the $\boldsymbol{k}=0$ level of the band at $20192 \mathrm{~cm}^{-1}$. On adding the fully deuterated isotopic impurity, linear cluster states are created and discrete spectra are now observed throughout the whole exciton band. These spectra can be fitted to 
theoretical formulae for cluster energies and statistical distributions, each formula involving a nearest neighbour resonance interaction parameter ( $V_{c}$ in this case). We have observed cluster states containing up to six molecules

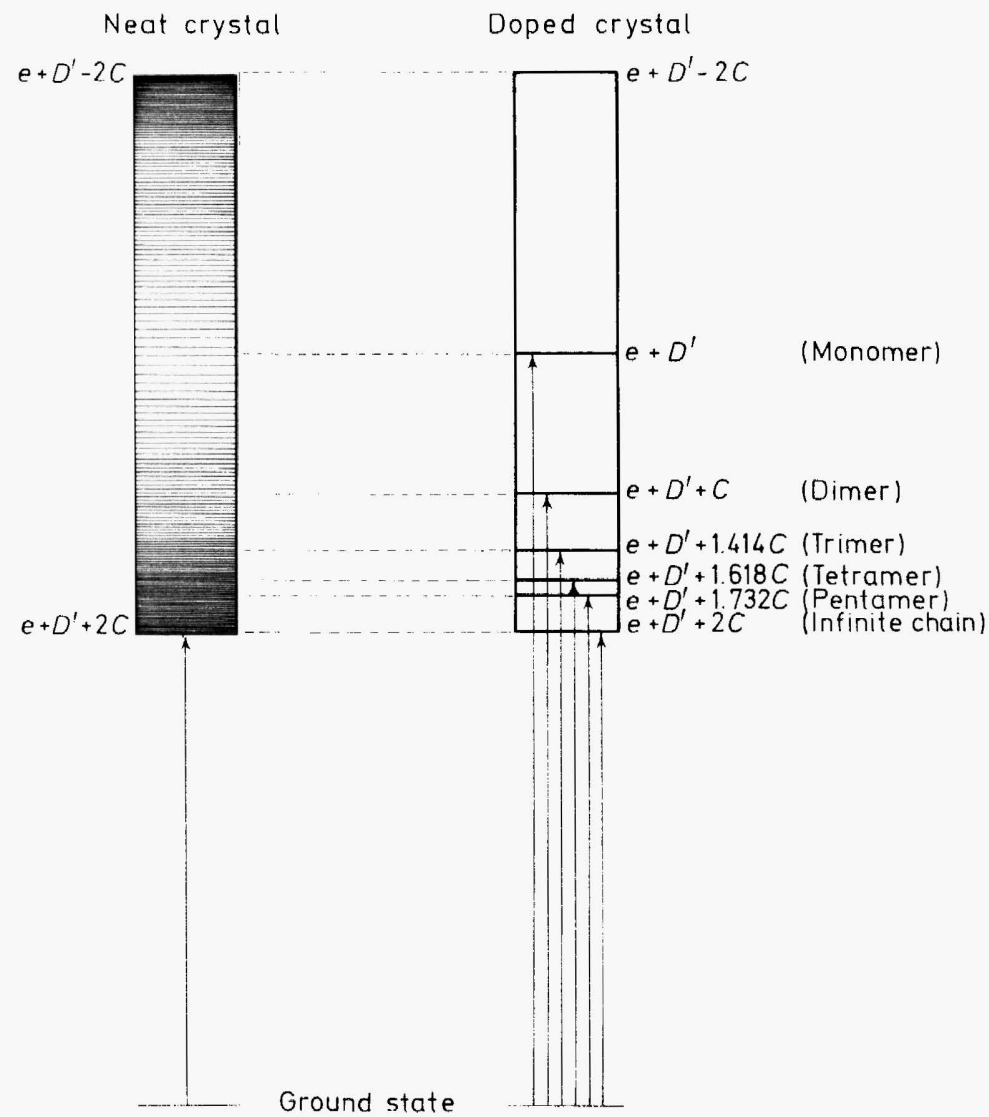

Figure 1. Schematic diagram of expected spectra of a heavily doped inverted linear chain band. Only the strongest transitions are depicted

and therefore we have six independent measurements of $V_{c}$. Each cluster state gave the same result for $V_{c}$ within the error quoted above so this study provides a confirmation of the nearest neighbour approximation, as well as establishing the linear chain nature of the band and the sign of the interaction parameter. The latter conclusion and our intuition regarding the $c$ direction was later confirmed by means of the exciton diffusion studies by $\operatorname{Ern}^{10}$ that show $D_{c}$ to be much larger than $D_{a}$ or $D_{b}$. Recently, optically detected e.p.r. studies have been used to estimate the much smaller interstack resonance interaction $^{12}$.

\section{LUMINESCENCE PROPERTIES OF LINEAR CHAIN SYSTEMS}

The luminescence of DBN neat crystal consists of transitions originating at many traps and of exciton emission. The lifetizhed 
R. M. HOCHSTRASSER, T.-Y. LI, H.-N. SUNG, J. WESSEL AND A. H. ZEWAIL

is about $0.5 \mathrm{~ms}$ at $1.6 \mathrm{~K}$. The effective dimensionality of the excitations in this crystal is dramatically evidenced by the nearly 100-fold increase in phosphorescence that arises when the protocrystal is doped with perdeutero molecules ${ }^{4}$. The luminescence of the mixed crystal comes from a variety of cluster states and the results give new information on the nature of energy transfer between clusters in these crystals.

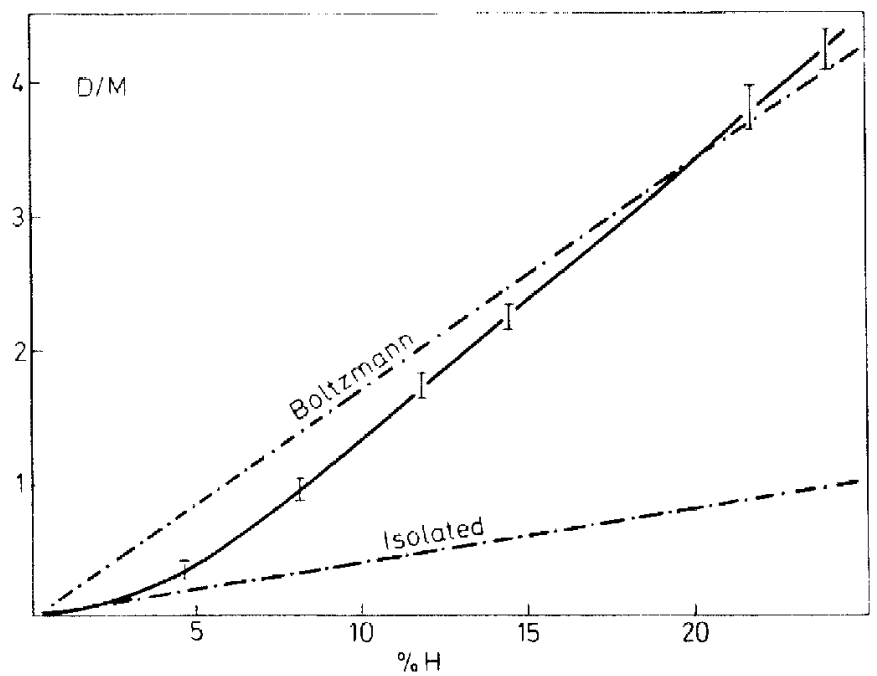

Figure 2. Variation with dibromonaphthalene $(D B N)-h_{6}$ in DBN- $d_{6}$ concentration of the ratio of dimer (D) to monomer (M) emission intensity at the 0,0-transition. The temperature is $4.2 \mathrm{~K}$.

In recent work by Zewail ${ }^{13}$ it was observed that at $4.2 \mathrm{~K}$ the Boltzmann distribution over the monomer and dimer states is only achieved when the concentration of DBN- $h_{6}$ in DBN- $d_{6}$ exceeded about 10 mole per cent. Preliminary results at low spectral resolution are shown in Figure 2 and their probable interpretation involves the sensitivity of the trap-to-trap migration ${ }^{14}$ of excitation to the distance between these cluster states. If the thermalizing mechanism in the mixed crystal involved only the intrachain interactions and if during the lifetime of the trapped excitation $(\tau \approx 4 \mathrm{~ms})$ the number of different clusters visited $(N)$ is determined by a one-dimensional random walk process, then:

$$
N \approx\left(\tau V_{c} / h\right)^{\dot{a}}\left(V_{c} / \Delta E\right)^{n i 2}
$$

where $n$ is the average number of host molecules between clusters and $\Delta E$ is the deuteration shift of $65 \mathrm{~cm}^{-1}$. In the case of DBN we have $\left(V_{\mathrm{c}} / \Lambda E\right) \approx$ 0.1 so $N$ is of order unity (i.e. the excitation remains on a few clusters) when $n \approx 8$. At concentrations of guests for which $n=4$ already $N=300$. Because of the mismatch in the monomer and dimer energy levels the intrachain trap-to-trap migration is slowed down so it is likely that interchain coupling participates in the thermalization mechanism. For $n=4$ the trap interaction 
energy is $\sim V_{c}^{n+1} / \Delta E^{n}$ which is about $7 \times 10^{-4} \mathrm{~cm}^{-1}$ and we may expect the interchain interaction to exceed this.

Since DBN molecules have no centre of inversion the two molecules A and $B$ in the translationally equivalent dimer are not equivalent so the wave function for the lower energy (emitting) dimer state in first-order must be written:

$$
\Psi_{\mathrm{D}}=C_{1} \varphi_{\mathrm{A}}^{\prime} \varphi_{\mathrm{B}}+C_{2} \varphi_{\mathrm{A}} \varphi_{\mathrm{B}}^{\prime}
$$

where $\varphi$ and $\varphi^{\prime}$ are Born-Oppenheimer states for different electronic excitation. We can tell that the excitation is close to being equally distributed over both molecules in the dimer from three observations. Firstly, the heavy doping experiments and computations of $V_{c}$ from these are self-consistent ${ }^{4}$. Zewail ${ }^{13}$ has shown that the ratio of dimer to monomer emission is roughly twice as large for the 0,0-transition as for emission on to vibrational levels of the ground state. This implies that $C_{1} \approx C_{2} \approx 1 / \sqrt{2}$ if the vibrational excitation is shared between the two molecules. The third observation that pertains to this question of delocalization of the excitation is the optically detected magnetic resonance of DBN monomers and dimers recently studied by Zewail ${ }^{13}$ in which averaging of ground and excited state coupling constants is observed in the dimer spectrum.

\section{BAND-TO-BAND TRANSITIONS}

Assuming that excitons thermalize in band states having definite $k$ vectors the luminescence from the neat crystal zero-point level band into bands corresponding to ground state vibrational levels should have the line shapes depicted in Figure 3 for various temperatures. These theoretical line shapes assume a vanishingly narrow vibrational exciton band and the line shape function is simply:

$$
g(\omega) \rho_{k}(\omega) \mathrm{e}^{-\omega_{k} i k T}
$$

where $g(\omega)$ is a Lorenzian. $\rho_{k}(\omega)$ is the density of $k$ states at $\omega_{k}=\epsilon_{k} / h$. Since we know the density of states in this case luminescence band-to-band experiments can be used to study the influence of exciton-phonon interactions on the radiative properties of molecular crystals. $\mathrm{Li}^{14}$ has studied band-toband transitions in DBN and a typical result is shown in Figure 4. We find the expected double humped emission line shape - the separation between the humps is somewhat less than $4 V$-proving that the excitons are really thermalized into definite $k$ states at these temperatures. Also the optical line shape for the zero-zero transition to the $\boldsymbol{k}=0$ region of the band is less than the exciton bandwidth indicating that the exciton-phonon scattering is not large enough to cause localization of the excitations. Nevertheless there seems to be an effect due to the finite width of the vibrational exciton bands of the ground state.

\section{EXCITON-PHONON COUPLING}

In considering exciton-phonon coupling in pseudo linear chain systems it is necessary to consider that the effective phonons may not be those of the 
chain and the optical phonons of the real crystal may play a part. Indeed temperature effects (Figure 4) on band-to-band transition may indicate

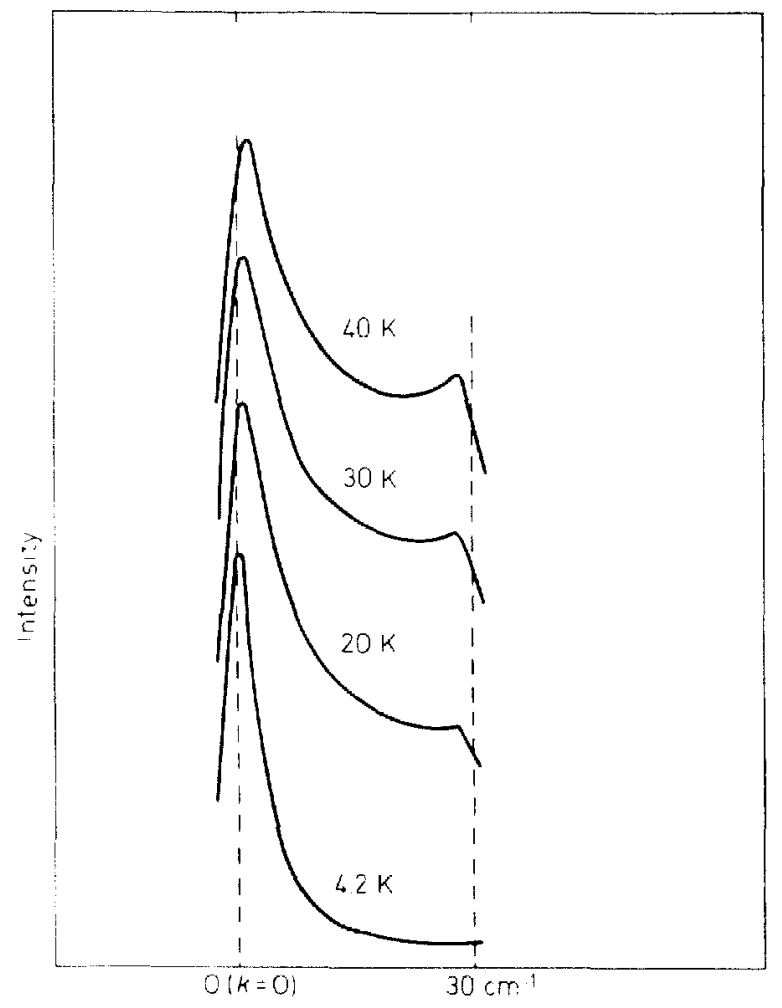

Figute 3. Calculated band $\left(\mathrm{T}_{1}\right)$ to band (vibrational) transition for linear chain

that population of phonon bands, other than a smoothly varying linear chain acoustic band, is occurring. The whole question of exciton-phonon coupling and its influence on exciton spectra and dynamics in molecular crystals is not yet fully explored. For example the absorption spectrum of DBN at low temperatures shows essentially no phonon sidebands, and we have proposed that this is because the exciton stabilization comes manly from the transfer of excitation energy in this case ${ }^{5}$. But this is not always so even in rather similar crystals, since in the case of dibromobenzene it appears as if the stabilization energy arises by an excited molecule changing its position in the lattice. The result is that intense phonon sidebands are seen in the absorption spectra just as if the excitations were small polarons ${ }^{5}$. In these crystals it is possible that the halogen halogen forces (attractive") determine the nature of the stacking of molecules: this would account for the quite similar contacts found in a variety of halogenated benzene crystals. The differences in exciton phonon coupling would derive from variations in the 
distance dependence of the van der Waals forces and it is not apparent why there should be other than small differences in a series of isomorphous (or 'nearly' isomorphous) structures. Again the interchain phonons have to be considered and so it is probably not justifiable to draw conclusions from the comparison of different structures (DBN and DBB) even though the $c$-chains have similar halogen contacts and interplanar separations.

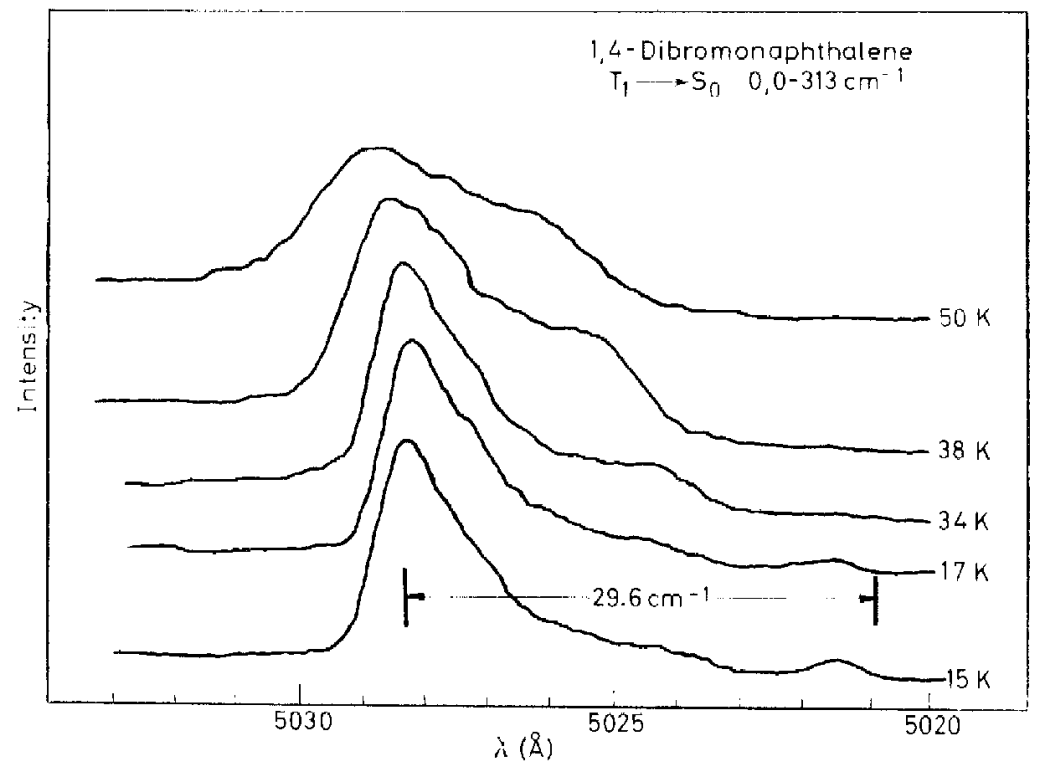

Figure 4. Emission of DBN- $\mathrm{h}_{5}$ at various temperatures in the region of the band-to-band energy corresponding to a $313 \mathrm{~cm}^{-1}$ mode of the ground state. The vertical bars indicatc the expected extremes based on the upper state being a linear chain of bandwidth $29.6 \mathrm{~cm}^{-1}$. Recent work by Zewail ${ }^{13}$ indicates that the actual bandwidth is close to $25 \mathrm{~cm}^{-1}$.

\section{BAND-STRUCTURE AND CARBON-13 TRANSITIONS}

Most of the halogenated benzene crystal spectra that have been studied show in addition to a strong sharp zero-phonon transition at least two additional weaker lines at $c a .4-6 \mathrm{~cm}^{-1}$ and $c a .711 \mathrm{~cm}^{-1}$ from the zerophonon line. These transitions were first observed by Castro ${ }^{16}$ but no interpretation was placed on them at that time. We later realized that they were most probably due to ${ }^{13} \mathrm{C}$ isotopic impurities ${ }^{9}$ in natural abundance. The six carbons of $p$-dichloro, $p$-dibromo and sym-tetrachloro-benzene (for example) group into two inequivalent sets containing four and two carbons. This grouping may account for the two weak lines seen in the singlet-triplet absorption spectra, and on that assumption it is possible to use these transitions to learn about details of the exciton band that may otherwise have been too minute to detect using optical spectroscopy.

For a zero-order trap depth of $\delta$, measured from the centre of the host exciton band, the trap energy $E$ for a linear chain is ${ }^{17,18}$

$$
E= \pm\left[4 V_{c}^{2}+( \pm) \delta^{2}\right]^{\frac{1}{2}}
$$


where the plus sign is used when $\delta$ is positive. The observed isotope transition always lies outside the band (i.e. $E>\left|2 V_{c}\right|$ ) and the intensity of transitions to the isotopic impurity is enhanced or diminished by the factor ${ }^{19}$ :

$$
\left(E+2 V_{c}\right)^{\frac{3}{2}} / E\left(E-2 V_{c}\right)^{\frac{1}{2}}
$$

It should be noted that the observed energy difference $\Delta \varepsilon_{i}$ between the assumed isotopic impurity line and the zero-phonon line of the host $(\boldsymbol{k}=0)$ is not equal to $E$. but is given by

$$
\Delta \varepsilon_{i}=E-2 V_{c}
$$

Thus a simple energy measurement in the neat and. say. chemically mixed crystal can measure $2 V_{c}$ for a linear chain. A plot of the quantity in equation 6 for $\Delta \varepsilon_{i}$ values of $5.2 \mathrm{~cm}^{-1}$ and $7.5 \mathrm{~cm}^{-1}$ is shown in Figure 5 and it can be seen that extremely narrow bandwidths can cause readily measurable effects on the ${ }^{13} \mathrm{C}$ transitions. As we learned many years ago (I herewith acknowledge helpful discussions with Professor T. M. Dunn during 1968 regarding the spectra of $p$-dichloro- and $p$-dibromobenzene) a high resolution spectrometer

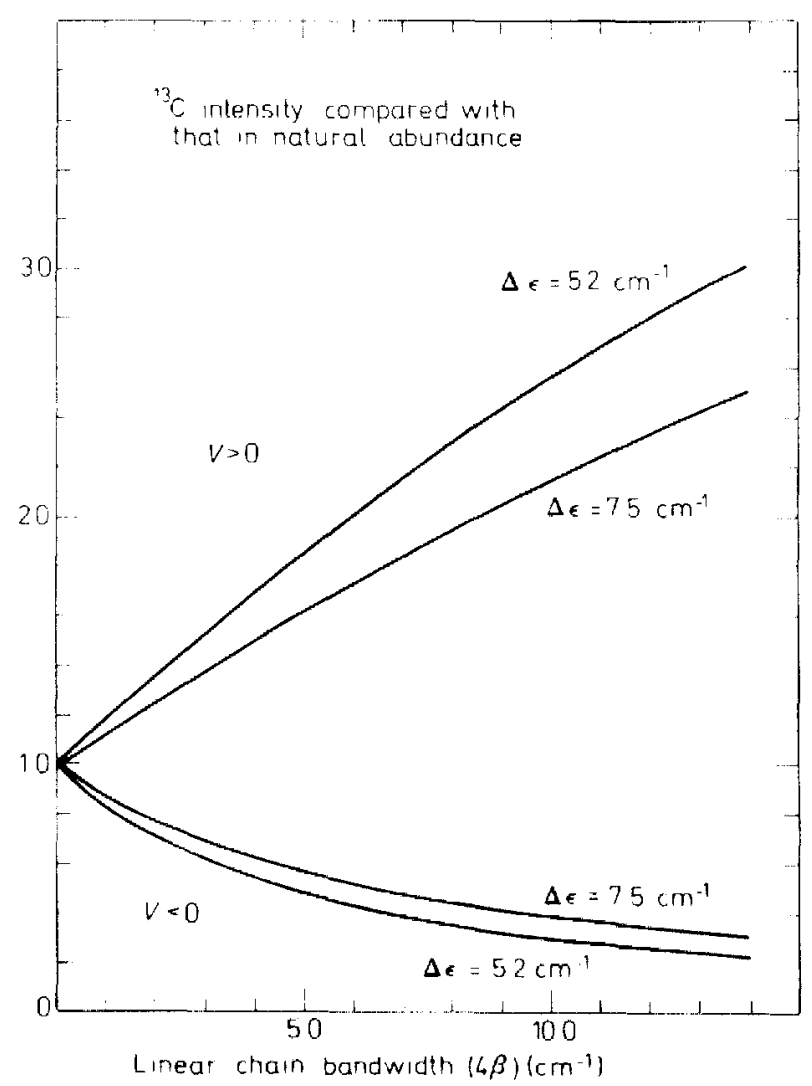

Figure 5. Theoretical relative intensity changes expected for shallow traps in a linear chain band 
must be used to measure the relative intensities of the zero-phonon lines since they may be exceedingly sharp- and our early intensity data ${ }^{17}$ were soon found to be unreliable because of a spectrometer limitation ${ }^{19}$. In the absence of site shifts the shift between a neat crystal transition and that transition in a dilute mixed crystal (proto in deutero) is equal to $2 V_{c}$ for a linear chain. For example in the case of tetrachlorobenzene the 0.0 -transition for the ncat crystal coincides $\left( \pm 0.2 \mathrm{~cm}^{-1}\right)$ with the dilute $h_{2}$ in $d_{2}$ origin indicating that if the system is a linear chain $2 V_{c}$ is less than $0.2 \mathrm{~cm}^{-1}$, assuming that the site shift is negligible. The ${ }^{1.3} \mathrm{C}$-band intensities are difficult to assess accurately for tetrachlorobenzene because the crystals are twinned triclinic and additional lines appear in the absorption spectrum near to the origin bands. The assumed ${ }^{13} \mathrm{C}$ transitions for tetrachlorobenzene molecules isolated in a dilute mixed crystal of durene appear at $5.5 \pm 0.2 \mathrm{~cm}^{-1}$ (weaker) and $7.7 \pm 0.2 \mathrm{~cm}^{-1}$ (stronger $\times 2$ ) to higher energy than the ${ }^{12} \mathrm{C}$ zero-zero transition. The intervals in the neat crystal are $5.7 \pm 0.3 \mathrm{~cm}^{-1}$ and $7.6 \pm$ $0.3 \mathrm{~cm}^{-1}$ with the former the stronger. If these pairs of levels are assumed to be the ${ }^{13} \mathrm{C}$ transitions in both neat and chemically mixed crystals, and if it is assumed that the site shifts for $\left({ }^{13} \mathrm{C}-\mathrm{Cl}\right) \mathrm{C}_{5} \mathrm{H}_{2} \mathrm{Cl}_{3}$ and $\left({ }^{13} \mathrm{C}-\mathrm{H}\right) \mathrm{C}_{5} \mathrm{HCl}_{4}$ are the same as for $\mathrm{C}_{6} \mathrm{H}_{2} \mathrm{Cl}_{4}$, then using equations 5 and 7 the bandwidth must satisfy the inequality:

$$
0<4 V_{c}<0.8 \mathrm{~cm}^{-1}
$$

in agreement with isotopically mixed crystal results.

\section{HEAVY DOPING AS BAND-STRUCTURE INDICATOR}

For one-dimensional and some two-dimensional bands the effects of energetically distant deutero molecules on the spectrum of the proto crystal is such that the band structure and therefore the exciton dimensionality can be exposed by doping experiments such as those used for $\mathrm{DBN}^{4}$ and pyrimidine ${ }^{12}$. Heavy doping is also useful for exposing narrow exciton bands. The quantity measured in a heavy doping experiment is the spectral density ${ }^{20}$ for $\boldsymbol{k}=0$ and for systems having simple dimensionality this distribution is wide enough for about one-half of the band to be exposed by conventional optical absorption studies (for example, see the DBN spectra from Ref. 4). In the case of the halogenated benzenes that display the ${ }^{13} \mathrm{C}$ transitions discussed above the effect of heavy doping is rather slight. Figure 6 shows the absorption spectrum of a 50 per cent mixed $p$-dibromobenzene- $h_{4}$ and $d_{4}$ crystal in the region of the zero-zero band. The asymmetric line broadening of the zero-phonon transitions is less than $c a .0 .5 \mathrm{~cm}^{-1}$ implying a linear chain bandwidth of less than $1 \mathrm{~cm}^{-1}$ : the absence of Davydov splitting $\left(<0.5 \mathrm{~cm}^{-1}\right)$ indicates that the interchange equivalent interaction is less than $0.5 / 8 \mathrm{~cm}^{-1}$.

Tetrachlorobenzene shows no asymmetric line broadening on heavy doping (see Figure 7), but there is a small $\left(c a .0 .5 \mathrm{~cm}^{-1}\right)$ symmetric line broadening. In this case the line width of the neat crystal transition is $2.0 \mathrm{~cm}^{-1}$ so if the system is a linear chain exciton the bandwidth is indicated to be less than $c a .2 \mathrm{~cm}^{-1}$, consistent with the mixed crystal energies discussed above, and with approximate values for the energies of the ${ }^{13} \mathrm{C}$ transitions based on a 
R. M. HOCHSTRASSER, T.-Y. LI, H.-N. SUNG, J. WESSEL AND A. H. ZEWAIL

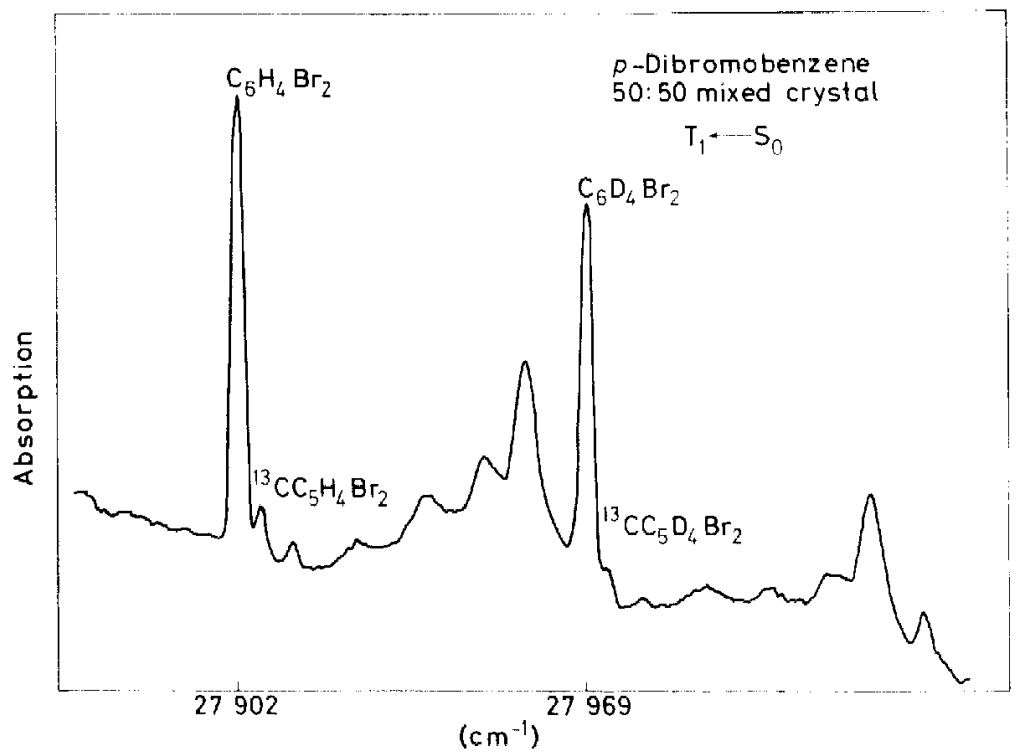

Figure 6. Absorption spectrum of a 50:50 mixed crystal of p-dibromobenzene- $h_{4}$ and $d_{4}$. Note the lack of line broadening and the similarity of ${ }^{13} \mathrm{C}$ transitions in the $h_{4}$ and $d_{4}$ spectra

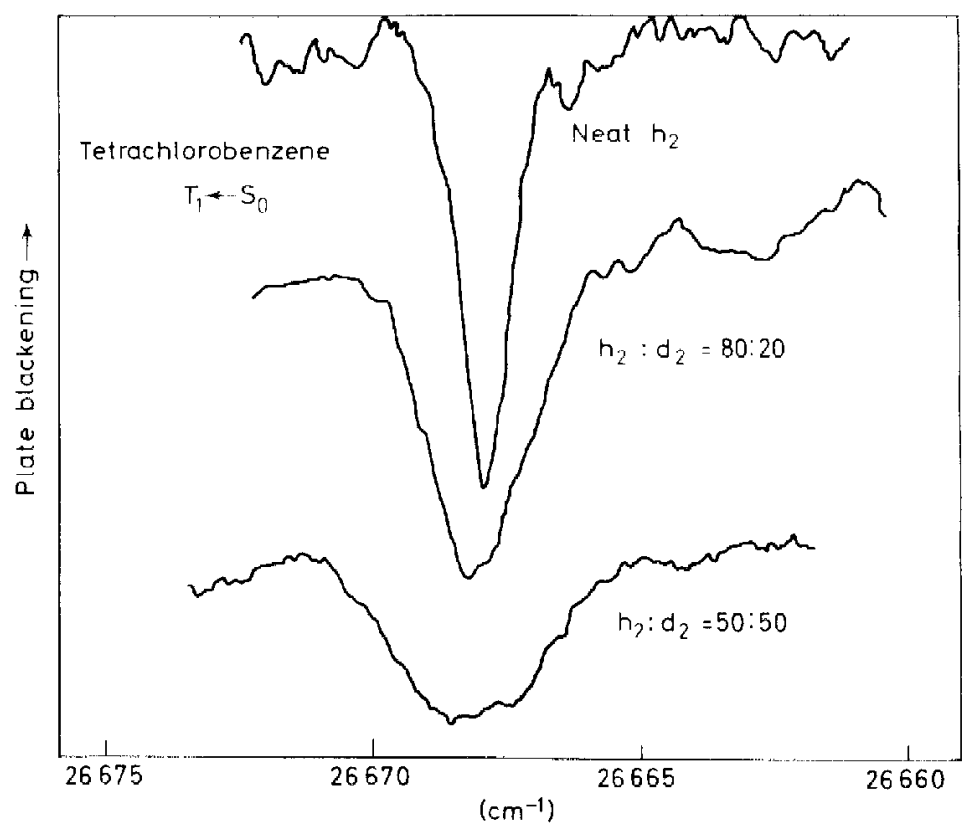

Figure 7. Heavy doping with tetrachlorobenzene- $\mathrm{d}_{2}$ of $\mathrm{TCB}-\mathrm{h}_{2}$. Note the symmetric line broadening 
linear chain exciton with $k=0$ at the top of the band as suggested by the experiments of Francis and Harris ${ }^{21}$. The bandwidth suggested by the optical experiments is narrower than that deduced by Harris. However we cannot tell whether or not tetrachlorobenzene is a linear chain; we can only put restrictions on the bandwidth of the exciton based on assumptions regarding its topology.

\section{TWO-PHOTON SPECTROSCOPY AND EXCITONS}

In concluding this discussion of triplet excitons I wish to mention a spectroscopic technique that Sung and Wessel have recently used to study

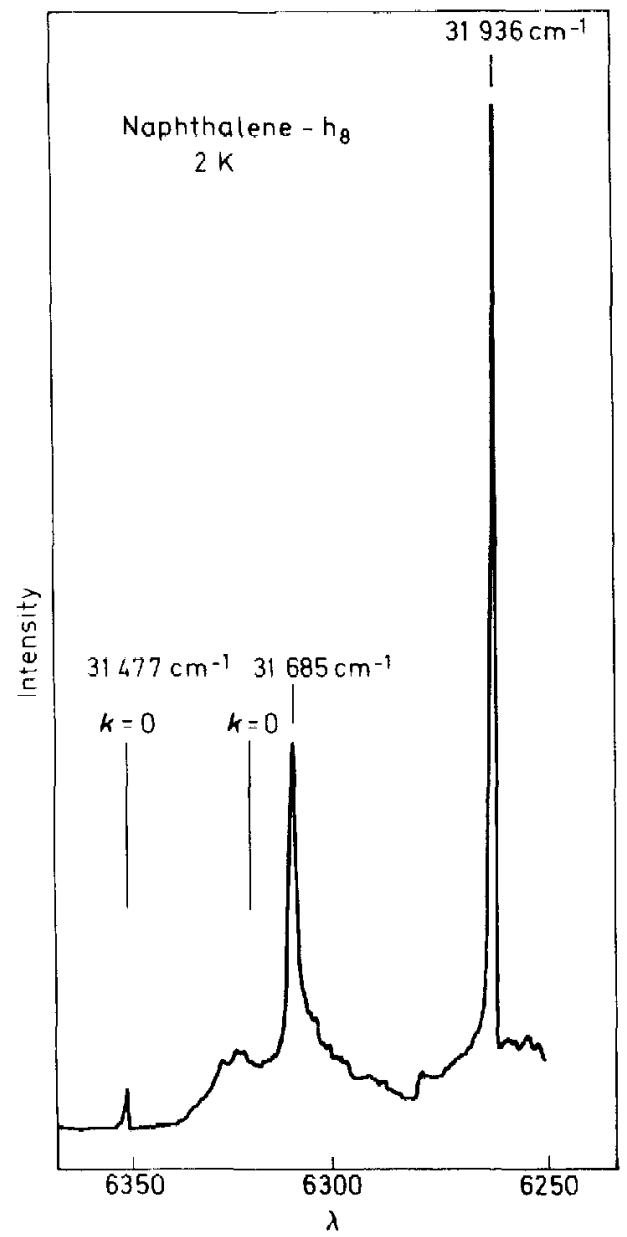

Figure 8. Two-photon unpolarized excitation spectrum of naphthalene in the neighbourhood of the 0,0 -band of the first singlet state. The vertical bars mark the positions of the $k=0$ exciton levels known from one-photon spectra 
singlet excitons, and which can be adapted to study triplets. The technique is high-resolution two-photon excitation spectroscopy 22 and involves scanning in the region of one-half the exciton energy with a tunable dye laser. A small portion of the spectrum of a single crystal of naphthalene at $1.6 \mathrm{~K}$ is shown in Figure 8 . The spectrum refers to the origin region of the first excited singlet state of the naphthalene crystal $^{23}$. The bars marked $\boldsymbol{k}=0$ refer to the positions of the $\boldsymbol{k}=0$ transitions in one-photon spectroscopy. The $31685 \mathrm{~cm}^{-1}$ band is not seen in the one-photon spectrum. Two aspects of these spectra are pertinent in the present context: the presence of the $31685 \mathrm{~cm}^{-1}$ line. which presumably has g parity, so close to the exciton band edge emphasizes the incompleteness of one-photon optical studies based on only u parity levels: the presence of u parity $k=0$ transitions that have essentially the same appearance as they have in the one-photon spectrum ${ }^{24}$ is quite a new phenomenon. We suggest that the $k=0$ transitions occur because of the simultaneous absorption of two photons, one via an electric quadrupole/magnetic dipole mechanism. The probability amplitude for such a process is low enough that we believe two-photon absorption studies of singlet-triplet transitions and of triplet excitons are now also within reach.

\section{REFERENCES}

1 G. Castro and R. M. Hochstrasser, Mol. Cryst., 1, 139 (1966).

2 W. T. Simpson and D. L. Petersen, J. Chem. Phys., 26. 588 (1957).

J. Jortner, S. A. Rice. J. L. Katz and S. I. Choi, J. Chem. Phys. 42, 309 (1965).

R. M. Hochstrasser and J. D. Whiteman, J. Chem. Phys.. 56, 5945 (1972).

R. M. Hochstrasser and P. Prasad, J. Chem. Phys., 56. 2814 (1972).

${ }^{6}$ R. H. Clarke and R. M. Hochstrasser, J. Chem. Phys. 46, 4532 (1967)

R. H. Clarke and R. M. Hochstrasser, J. Chem. Phys., 49, 3313 (1968)

* G. W. Robinson, Ann. Rev. Phys. Chem., $21.429(1970)$.

${ }^{9}$ R. M. Hochstrasser and J. W. Whiteman, (a) 24th Symposium on Molecular Structure and Spectroscopy, Abstract I.11, Columbus, Ohio (1969): (b) Organic Scintillators and Liquid Scintillation Counting, p.51 J, (ed. D. L. Horrocks and C..-T. Peng), Academic Press. New York (1971), (proceedings of a conference in San Francisco, July 1970): (c) 26th Symposium on Molecular Structure and Spectroscopy, Abstract U1, Columbus, Ohio (1971).

10 V. Ern, J. Chem. Phys., 56, 6259 (1972).

11 R. Schmidberger and H. C. Wolf, Chem. Phys. Letters, 16, 402 (1972).

12 J. D. Whiteman, PhD Dissertation. University of Pennsylvania (1971).

13 R. M. Hochstrasser and A. H. Zewail, J. Chem. Phys., in press

14 II. Sternlicht. G. C. Nieman and G. W. Robinson, J. Chem. Phys. 38, 1326 (1963).

R. M. Hochstrasser and T.-Y. Li, to be published.

if G. Castro and R. M. Hochstrasser, J. Chem. Phys. 46, 3617 (1967).

17 G. F. Koster and J. C. Slater, Phys. Rev., 95, 1167 (1954).

1 " D. P. Craig and M. R. Phillpott, Proc. Roy. Soc. (London). A243, 213 (1966).

${ }^{19}$ See Ref. 5, footnote 32.

2) P. Soven, Phys. Rev.. 178, 1136 (1969)

${ }^{2}$ C. B. Harris, proceedings of this conference, this volume, p. 73; see also $\Lambda$. H. Francis and

C. B. Harris, Chem. Phys. Letters, 9, 181 (1971).

22 R. M. Hochstrasser, H.-N. Sung and J. Wessel, J. chem. Phys., 58, 4694 (1973).

23 R. M. Hochstrasser, H.-N. Sung and J. Wessel, Chem. Phys. Letters, 24. I68 (1974).

24 See for example, S. D. Colson, D. M. Hanson, R. Kopelman and G. W. Robinson, J. Chem. Phys., 48, 2215 (1968). 